\title{
Cutaneous/Nasal Ointment Dosage Form
}

National Cancer Institute

\section{Source}

National Cancer Institute. Cutaneous/Nasal Ointment Dosage Form. NCI Thesaurus.

Code C149422.

Semi-solid preparation consisting of an ointment intended for cutaneous or nasal use. 